Research Article

\title{
The Stability of Magnetic Levitation Milling System Based on Modal Decoupling Control
}

\author{
Xiaoli Qiao (iD) and Xiaoping Tang \\ Shaoxing University Yuanpei College, Shaoxing 312000, China \\ Correspondence should be addressed to Xiaoli Qiao; qiaoxiaoli168@163.com
}

Received 6 July 2019; Revised 17 September 2019; Accepted 18 September 2019; Published 19 May 2020

Academic Editor: Chao Tao

Copyright (c) 2020 Xiaoli Qiao and Xiaoping Tang. This is an open access article distributed under the Creative Commons Attribution License, which permits unrestricted use, distribution, and reproduction in any medium, provided the original work is properly cited.

\begin{abstract}
Milling stability not only reduces the surface quality of the workpiece but also seriously restricts the high-speed development of $\mathrm{CNC}$ machine tools. The electric spindle rotor system with the active magnetic bearing has a strong gyro coupling effect, and with the increasing rotor speed, it will become a major unfavorable factor for the stability of the system during high-speed milling. The strong gyro coupling effect makes the stability region narrow at the time of high-speed milling. So, a modal decoupling control method that can reduce the effects of the gyro effect on the magnetic levitation milling system under high-speed milling is proposed. The effects of the gyro coupling of the magnetic bearing rotor on the milling stability region before and after the decoupling control are studied, which show that the modal decoupling control technology can reduce the effects of the gyro effect on the magnetic levitation milling system.
\end{abstract}

\section{Introduction}

The high-speed milling is the only way to improve milling efficiency, and the magnetic suspension support technology is one of the world's recognized high technology and, of course, the first choice for high-speed milling machines. The high-speed magnetic suspension electric spindle rotor system has a strong gyro coupling effect at high speed, which will greatly affect the stability of the high-speed machining system and then affect the efficiency of milling processing and product quality. When the electric spindle rotor system is treated as the rigid rotor system, the modes of rotation and translation motion will occur. When the rotor of the electric spindle rotates at high speed, the rotational mode can be divided into positive precession mode and opposite precession mode under the action of the strong gyro coupling effect of the rotor. In the actual magnetic suspension electric spindle rotor system, the bandwidth of the power amplifier and the sensor is limited, which will lead to a time delay of the electromagnetic force, and the force produced by the magnetic bearing will put energy into the electric spindle rotor; the energy of the opposite precession mode will be put into the positive feedback and be accumulated, which will lead to a unstable milling system. In addition, the damping effect of the control force will also decrease as the precession modal frequency decreases; especially, when the controller includes the integral part, the phase advance of the low frequency band is difficult to guarantee. When the precession mode frequency finally enters the range in which the integral parameter acts, the precession mode will also cause the milling system to be unstable [1].

In order to reduce the gyro coupling effect of the highspeed magnetic suspension electric spindle rotor system on milling stability, it is necessary to suppress the positive precession mode and the opposite precession mode generated by the gyro coupling effect. In this regard, various solutions have been proposed, mainly including control methods based on modern control theory, such as sliding mode control [2], $\mu$ synthesis [3], gain-scheduled $H \infty$ control [4], Cholesky decomposition reduction [5], and LQR control [6]. Although these control methods had played a role in suppressing the gyro effect, these algorithms are complex and computationally intensive and are difficult to implement in engineering due to hardware conditions. 
There is also a cross-feedback control method based on the traditional PD controller, in which the cross-feedback can be divided into displacement crossover [7], velocity crossover, velocity and displacement crossover [8], and displacement crossover combined with electromagnetic force [9]. The advantage of cross-feedback, especially the speed cross-feedback method, is that the structure is simple and is easy to be implemented. The disadvantage is that there is still no effective cross-feedback parameter design method. In addition, when the traditional PD control is used, the characteristics of each mode are difficult to be independently adjusted because there is the strong coupling between the rotational mode and the translational mode of the magnetic electric spindle rotor. Therefore, the control methods based on this have certain limitations.

In view of this, based on the mathematical model of the active magnetic bearing electric spindle rotor system, a modal decoupling control method is proposed, which can decouple the rotating mode and the translational mode of the active magnetic bearing electric spindle rotor system, in order to control the stiffness and damping of the translational mode and the rotation mode independently, which can reduce effectively the influence of the gyro effect on the high-speed milling stability area and then improve the stability area of the highspeed magnetic suspension electric spindle milling system.

\section{Mathematical Model of Four-Degree-of- Freedom Magnetic Suspension Electric Spindle Rotor System}

The structure of the magnetic bearing rigid electric spindle rotor system is shown in Figure 1. Ideally, the axis of the rotor coincides with the center of the two radial bearings. In order to describe the mutual position between the rotor, the sensor, and the electromagnetic bearing, the main coordinate system oxyz is set up, in which the coordinate origin is at the centroid point $c$ of the rotor, and The $z$-axis coincides with the center line of the two radial bearings. The distance from the center of the left and right radial electromagnetic bearings $A$ and $B$ to point $c$ is, respectively, $l_{m A}$ and $l_{m B}$, and $l_{m A}$ is a negative number, while $l_{m B}$ is a positive number. The distances of the left and right sensors $A$ and $B$ to $c$ are, respectively, $l_{s A}$ and $l_{s B} ; l_{s A}$ is negative, and $l_{s B}$ is positive. In order to make the analysis easy, three coordinate systems of radial planes are established, which are the sensor coordinate system, the active magnetic bearing coordinate system, and the centroid coordinate system; the coordinate origin is set on the center line of the two radial bearings. When the rotor rotates, the coordinates at the center of mass of the rotor are $x, y, \theta_{x}$, and $\theta_{y}$.The coordinates at the left and right sensors are $x_{s A}, x_{s B}, y_{s A}$, and $y_{s B}$, and the coordinates of the left and right active magnetic bearings are $x_{m A}, x_{m B}, y_{m A}$, and $y_{m B}$, respectively. The ignorance of the sensor and magnetic bearing installation position error can make $x_{s A}=x_{m A}=x_{a}$, $x_{s B}=x_{m B}=x_{b}, \quad y_{s A}=y_{m A}=y_{a}, \quad y_{s B}=y_{m B}=y_{b}$, $l_{s A}=l_{m A}=l_{a}, l_{s B}=l_{m B}=l_{b}$, and $l=l_{s A}-l_{s B}$. When the displacement of the rotor is $x_{a}, y_{a}, x_{b}$, and $y_{b}$ in the $x_{a}, y_{a}$, and $y_{b}$ in the $y, \alpha, \beta$, and directions, the displacement of the rotor geometric center $O$ is

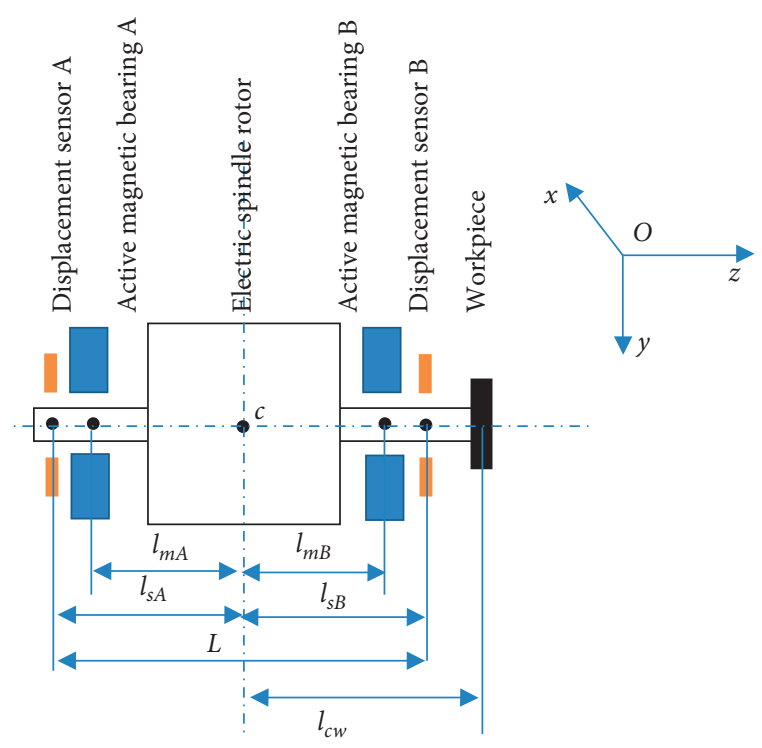

FIgURE 1: Active magnetic bearing-supported motorized spindle milling system.

$$
\left\{\begin{array}{l}
x=\frac{l_{b}}{L} x_{a}-\frac{l_{a}}{L} x_{b} \\
y=\frac{l_{b}}{L} y_{a}-\frac{l_{a}}{L} y_{b}
\end{array}\right.
$$

The angle at which the rotor rotates counterclockwise about the $x$-axis is

$$
\theta_{x}=\frac{y_{b}-y_{a}}{L}
$$

The angle at which the rotor rotates counterclockwise around the $y$-axis is

$$
\theta_{y}=\frac{x_{a}-x_{b}}{L}
$$

When the motion equation of the four-degree-of-freedom electric spindle rotor system with active electromagnetic bearing is established, since the bearing air gap is small, it can be assumed that (1) the rotor is an axisymmetric rigid rotor; that is, the moments of inertia around the $x$ and $y$ axes are equal. (2) The structure and parameters of the radial fourdegree-of-freedom are exactly the same; (3) the magnetic field in the electromagnet is evenly distributed, the magnetic flux leakage is ignored, and the magnetic material does not exhibit saturation characteristics; (4) the influence of the magnetic resistance and loss of the core material are ignored. Without considering the influence of external damping factors, it is easy to establish the motion equation of the axially symmetric active magnetic bearing electric spindle rotor system according to the rotor dynamics theory [10]:

$$
\left\{\begin{array}{l}
m \ddot{x}=F_{m x A}+F_{m x B}, \\
m \ddot{y}=F_{m y A}+F_{m y B}, \\
J \ddot{\theta}_{y}-J_{z} \omega \dot{\theta}_{x}=F_{m x A} l_{a}-F_{m x B} l_{b}, \\
J \ddot{\theta}_{x}-J_{z} \omega \dot{\theta}_{y}=-F_{m y A} l_{a}+F_{m y B} l_{b},
\end{array}\right.
$$


where $m$ is the electric spindle rotor quality, $J_{x}=J_{y}=J$ is the rotor moment inertia around $x$ and $y$ axis, and $y_{z}$ is the rotor moment inertia around $z$ axis. $F_{m x A}, F_{m x B}, F_{m y A}$, and $F_{m y B}$ are, respectively, the electromagnetic force of the end $A$ and end $B$ in the direction of $x$ and $y$ of the active electromagnetic bearings.

Equation (4) is transformed into a matrix:

$$
\text { Here, } M=\left[\begin{array}{cccc}
J & 0 & 0 & 0 \\
0 & m & 0 & 0 \\
0 & 0 & J & 0 \\
0 & 0 & 0 & m
\end{array}\right] \text { is the mass matrix of the rotor }
$$

system, $G=\left[\begin{array}{cccc}0 & 0 & J_{z} \Omega & 0 \\ 0 & 0 & 0 & 0 \\ -J_{z} \Omega & 0 & 0 & 0 \\ 0 & 0 & 0 & 0\end{array}\right]$ is the gyro effect matrix of the rotor system, $L_{f}=\left[\begin{array}{cccc}l_{a} & -l_{b} & 0 & 0 \\ 1 & 1 & 0 & 0 \\ 0 & 0 & -l_{a} & l_{b} \\ 0 & 0 & 1 & 1\end{array}\right]$ is force arm coefficient matrix, $F=\left(F_{m x A}, F_{m x B}, F_{m y A}, F_{m y B}\right)^{T}$ is the electromagnetic force vector of the active magnetic bearing, and $q=\left(\theta_{y}, x, \theta_{x}, y\right)^{T}$ is the coordinate of the centroid of the rigid electric spindle.

The electromagnetic force generated by the active magnetic bearing is a quadratic function of the coil current and the air gap between the stator and rotor [10], as follows:

$$
F_{m}=\frac{\mu_{0} A_{a} N^{2}}{4} \frac{i^{2}}{\delta^{2}}
$$

where $\mu_{0}$ is air permeability, $A_{a}$ is the cross-sectional area of the iron core and air gap, $N$ is the number of turns, $i$ is the coil current, and $\delta$ is the length of the air gap.

When the structure parameter is constant and the rotor moves in a small range near its static equilibrium position, equation (6) can be expanded as Taylor at the static equilibrium point, and the high-order small amount is omitted. The electromagnetic force can be expressed as a linear function of the current stiffness coefficient and the displacement stiffness coefficient [12]. Since the rotor is placed vertically, the bias currents on all the poles can be the same. The electromagnetic force can be expressed as

$$
\begin{aligned}
F & =\left[\begin{array}{l}
F_{m x A} \\
f_{m x B} \\
f_{m y A} \\
f_{m y B}
\end{array}\right]=\left[\begin{array}{cccc}
k_{s} & 0 & 0 & 0 \\
0 & k_{s} & 0 & 0 \\
0 & 0 & k_{s} & 0 \\
0 & 0 & 0 & k_{s}
\end{array}\right]\left[\begin{array}{l}
x_{a} \\
x_{b} \\
y_{a} \\
y_{b}
\end{array}\right]+\left[\begin{array}{cccc}
k_{i} & 0 & 0 & 0 \\
0 & k_{i} & 0 & 0 \\
0 & 0 & k_{i} & 0 \\
0 & 0 & 0 & k_{i}
\end{array}\right]\left[\begin{array}{l}
i_{m x a} \\
i_{m x b} \\
i_{m y a} \\
i_{m y b}
\end{array}\right] \\
& =K_{s} q_{s}+K_{i} i .
\end{aligned}
$$

Here, $q_{s}=\left(x_{a}, x_{b}, y_{a}, y_{b}\right)^{T}$ is the displacement of the active magnetic bearing relative to the equilibrium point, $i=$ $\left(i_{m x a}, i_{m x b}, i_{m y a}, i_{m y b}\right)^{T}$ is control current, $K_{s}=\operatorname{diag}\left(k_{s}\right.$, $\left.k_{s}, k_{s}, k_{s}\right)$ is the matrix of electromagnetic bearing displacement stiffness coefficient, and $K_{i}=\operatorname{diag}\left(k_{i}, k_{i}, k_{i}, k_{i}\right)$ is the matrix of the current stiffness coefficient of the electromagnetic bearing.
After the structural parameters of the electromagnetic bearing, the current and the gap at the operating point are determined; the current stiffness coefficient and the displacement stiffness coefficient of the electromagnetic bearing are both constant.

Putting equation (7) into equation (5), the following equation is obtained:

$$
M \ddot{q}+G \dot{q}=L_{f}\left(K_{s} q_{s}+K_{i} i\right) .
$$

In order to unify the coordinate system, the sensor coordinate system $q_{s}$ on the right side of equation (8) is converted into a centroid coordinate system $q$. The coordinate conversion relationship can be obtained as follows:

$$
\left[\begin{array}{l}
x_{a} \\
x_{b} \\
y_{a} \\
y_{b}
\end{array}\right]=\left[\begin{array}{cccc}
l_{a} & 1 & 0 & 0 \\
-l_{b} & 1 & 0 & 0 \\
0 & 0 & -l_{a} & 1 \\
0 & 0 & l_{b} & 1
\end{array}\right]\left[\begin{array}{c}
\theta_{y} \\
x \\
\theta_{x} \\
y
\end{array}\right] .
$$

That is,

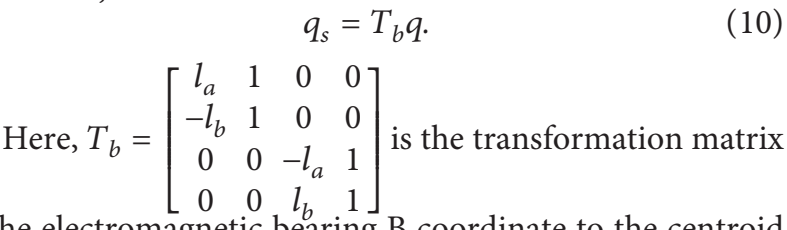
of the electromagnetic bearing $\mathrm{B}$ coordinate to the centroid coordinate.

The matrix $T_{b}$ is the transpose of the matrix $L_{f}$ :

$$
T_{b}=L_{f}^{T} \text {. }
$$

Putting equations (9) and (11) into equation (8), the following equation is obtained:

$$
M \ddot{q}+G \dot{q}=L_{f} K_{s} L_{f}^{T} q+L_{f} K_{i} i .
$$

Then, $K_{s s}=L_{f} K_{s} L_{f}^{T}$, and the following equation can be obtained:

$$
M \ddot{q}+G \dot{q}-K_{s s} q=L_{f} K_{i} i .
$$

Here,

$$
\begin{gathered}
K_{s s}=\left[\begin{array}{cccc}
k_{s}\left(l_{a}^{2}+l_{b}^{2}\right) & k_{s}\left(l_{a}-l_{b}\right) & 0 & 0 \\
k_{s}\left(l_{a}-l_{b}\right) & 2 k_{s} & 0 & 0 \\
0 & 0 & k_{s}\left(l_{a}^{2}+l_{b}^{2}\right) & k_{s}\left(-l_{a}+l_{b}\right) \\
0 & 0 & k_{s}\left(-l_{a}+l_{b}\right) & 2 k_{s}
\end{array}\right] . \\
\text { Then, } K_{s s}^{T}=\left(L_{f} K_{s} L_{f}^{T}\right)^{T}=L_{f} K_{s} L_{f}^{T}=K_{s s} .
\end{gathered}
$$

\section{Modal Decoupling Principles}

The purpose of the modal decoupling controller is to achieve the independent control between the rotational mode and the translational modal of the electromagnetic suspension spindle system. Firstly, the displacement signal at the four sensors of the input modal decoupling controller is converted into the translational displacement signal and the rotational angle signal at the center of mass of the rotor. That 
is, the coordinates of the sensor coordinate system $x_{a}, x_{b}, y_{a}$, and $y_{b}$ are converted into the coordinates of the centroid coordinate system $x, y, \theta_{x}$, and $\theta_{y}$, In this way, the rotor modes can be controlled independently. The PD control algorithm is still used in the modal decoupling controller to produce control signal.

Since the action point of control signal is at the mass center of the rotor, the actual electromagnetic bearing is located at both ends of the rotor; it is also necessary to convert the control signal at the center of mass of the rotor into a control signal of the electromagnetic force at the left and right electromagnetic bearings. That is, the coordinates of the centroid coordinate system are converted into the coordinates of the bearing coordinate system. Finally, the obtained electromagnetic force control signal together with the negative stiffness compensation signal is added to the power amplifier, which will generate the control current to control the stability of the milling process [13].

The control flow is shown in Figure 2.

The specific process of the modal decoupling controlled and its mathematical expression is designed. As shown in Figure 1, if $l_{m A} \neq-l_{m B}$, the distance between the A and $\mathrm{B}$ electromagnetic bearings to the origin of the coordinates is different, and the negative stiffness matrix $K_{s s}$ is not a diagonal matrix, which will weaken the effect of modal decoupling. After the translation of the centroid of the rotor, an electromagnetic force of the same magnitude is generated at the bearing positions. If it is an asymmetrical bearing, a nonzero torque is generated with respect to the centroid $c$, which will cause the rotor to rotate. In order to make the proportional and differential parameters of the PD controller act more directly on the modal stiffness, the negative stiffness $K_{s s}$ must be compensated.

If the current signal input to the power amplifier is divided into a current $i_{c}$ of the modal decoupling controller and a negative stiffness compensation current $i_{k}$, the current signal $i$ which will be input to the power amplifier by the controller is

$$
i=i_{c}+i_{k}
$$

Putting equation (15) into equation (13), the following equation can be obtained:

$$
M \ddot{q}+G \dot{q}+K_{s s} q=L_{f} K_{i} i_{c}+L_{f} K_{i} i_{k}
$$

As shown in equation (16), in order to make equation $K_{s s} q=L_{f} K_{i} i_{k}$ established, it is necessary to compensate the negative stiffness. Putting equation (10) into $K_{s s} q=L_{f} K_{i} i_{k}$, $i_{k}$ can be expressed as

$$
i_{k}=K_{i}^{-1} B^{-1} K_{s s} L_{s}^{-1} q_{s} .
$$

What will be discussed is the expression of the current signal $i_{c}$ of the modal decoupling controller. For the input of the controller, the output current signal by the conventional distributed PD controller is

$$
i_{c}=-P q_{s}-D \dot{q}_{s}
$$

Here,$P=\operatorname{diag}\left(p_{x A}, p_{x B}, p_{y A}, p_{y B}\right)$ is the proportional coefficient matrix and $D=\operatorname{diag}\left(d_{x A}, d_{x B}, d_{y A}, d_{y B}\right)$ is a differential coefficient matrix.

It can be known from equation (15) that the input change of any one control channels of the controller will cause the change in the rotation angle and the displacement of the center of mass of the rotor, which makes the rotational mode and the displacement mode coupled to each other at the input end. Therefore, the coordinates, $x_{s A}, x_{s B}, y_{s A}$, and $y_{s B}$ of the sensor coordinate system, should be converted into the coordinates of the centroid coordinate system, $x, y, \theta_{x}$, and $\theta_{y}$; that is, the matrix $q_{s}$ should be converted into a matrix $q$. The previous deduction leads to $q=\left(L_{f}^{T}\right)^{-1} q_{s}$, which means a coordinate transformation link with a relation matrix $\left(L_{f}^{T}\right)^{-1}$ is added before the sensor and the $\mathrm{PD}$ controller. In the physical sense, the direct adjustment of the modal offset signals by the PD control algorithm is accomplished.

For the output, the expression $L_{f} K_{i}$ is.

$$
L_{f} K_{i}=\left[\begin{array}{cccc}
K_{i} l_{b A} & K_{i} l_{b B} & 0 & 0 \\
K_{i} & K_{i} & 0 & 0 \\
0 & 0 & K_{i} l_{b A} & K_{i} l_{b B} \\
0 & 0 & K_{i} & K_{i}
\end{array}\right] .
$$

It can be seen that since $L_{f} K_{i}$ is not a diagonal matrix, there is a coupling between the rotational mode and the translational mode. In order to decouple the rotational mode and the translational mode at the output, it is also necessary to set a transformation matrix $T$ to make $L_{f} K_{i} T$ converted into a diagonal matrix. There are many different expressions for this kind of diagonal matrix. If $L_{f} K_{\mathrm{i}} T=I$, the matrix $T$ is obtained:

$$
T=K_{i}^{-1} L_{f}^{-1}=\frac{1}{k_{i}\left(l_{b A}-l_{b B}\right)}\left[\begin{array}{cccc}
-1 & l_{m B} & 0 & 0 \\
1 & -l_{m A} & 0 & 0 \\
0 & 0 & -1 & l_{m A} \\
0 & 0 & 1 & -l_{m A}
\end{array}\right] \text {. }
$$

From the physical point of view, modal decoupling control algorithm can directly adjust the modal of the rotor after the decoupling of the input end, but the output control signal, at this time for on the center of mass of the rotor, is the signal required for the stability of the rotor, not the electromagnetic force signal required for the two radial bearings. Therefore, a conversion link must be provided to convert the torque signal into the electromagnetic force signal of the electromagnetic bearing, in which way the decoupling between the input and output of the controller is realized, and finally, the current command signal of the PD controller is obtained as follows:

$$
i_{c}=-K_{i}^{-1} L_{f}^{-1}(P+D)\left(L_{f}^{T}\right)^{-1} q_{s} .
$$

Combining equations (15), (18), and (21), the following equation is obtained: 


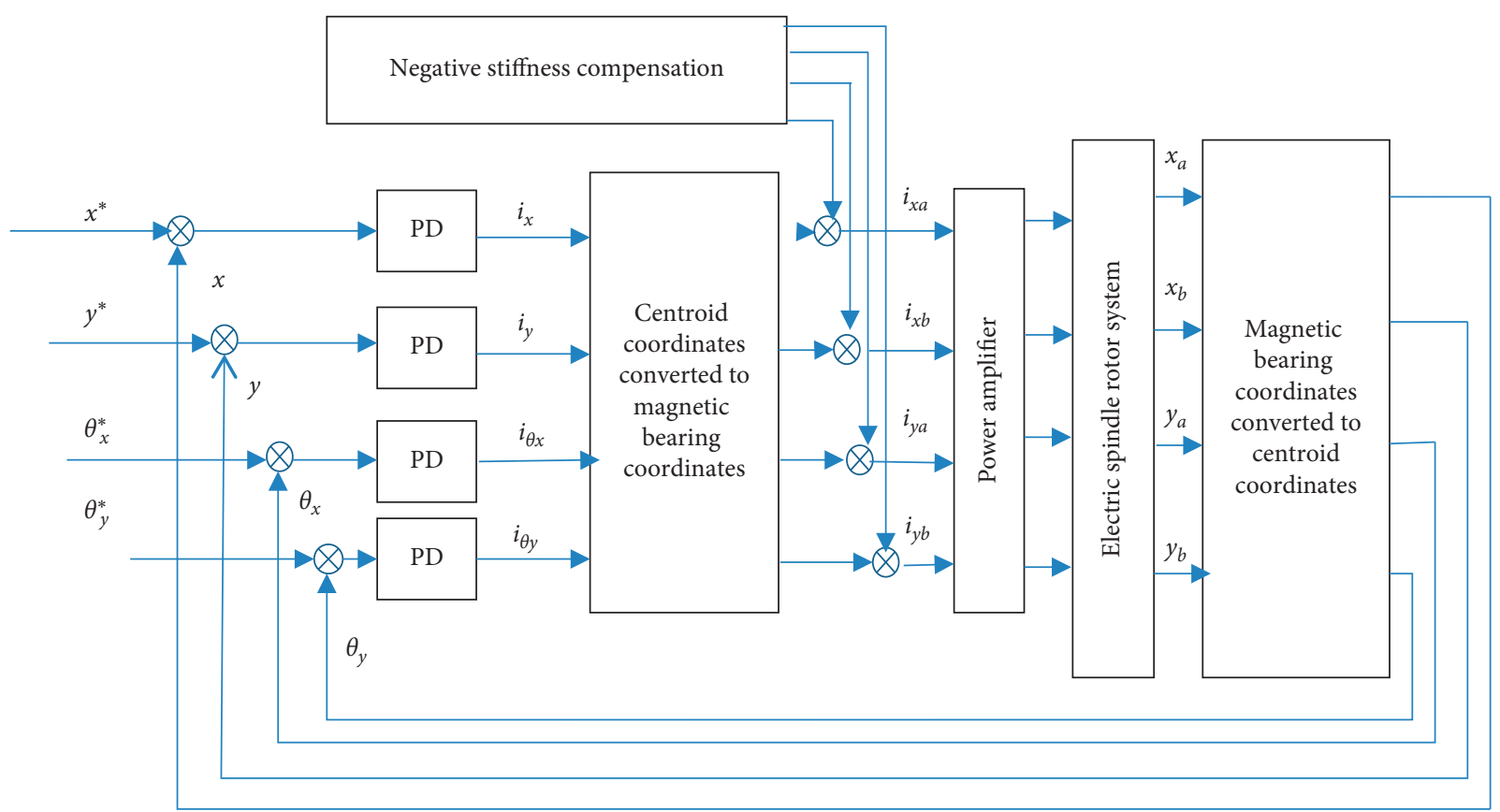

FIGURE 2: Modal decoupling control system.

$$
i=i_{c}+i_{k}=-K_{i}^{-1} L_{f}^{-1}\left(P+D-k_{s s}\right)\left(L_{f}^{T}\right)^{-1} q_{s} .
$$

Putting equations (19) and (22) into equation (8), then

$$
M \ddot{q}+G \dot{q}+P q+D \dot{q}=0,
$$

$$
\begin{aligned}
& P=\operatorname{diag}\left(p_{r}, p_{p}, p_{r}, p_{p}\right), \\
& D=\operatorname{diag}\left(d_{r}, d_{p}, d_{r}, d_{p}\right),
\end{aligned}
$$

where $p_{r}$ and $d_{r}$ are, respectively, the proportional and differential coefficients in the rotational mode control channel, while $p_{p}$ and $d_{p}$ are, respectively, the proportional and differential coefficients in the translational mode control channel.

It can be seen from equations (24) and (25) that the translational mode and the rotational mode control channel are independent of each other so that the stiffness and damping of each mode can be adjusted by changing $p_{r}, p_{p}$, $d_{r}$, and $d_{p}$. In addition, the gyro matrix $G$ only has a coupling effect on the rotating modal control channel and no influence on the translational modal control channel, and the gyro coupling effect is significantly reduced.

\section{Influence of Cross-Coupling Effect of Active Magnetic Bearing Gyro on Grinding Stability}

4.1. Dynamic Milling Force Model. The dynamic milling process with four cutter cylindrical end mills is shown in Figure 3 [11].

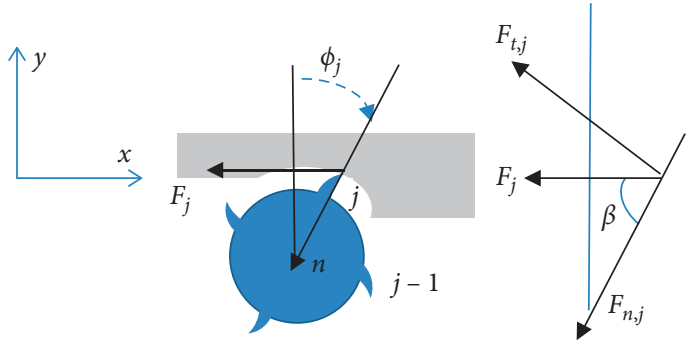

FIGURE 3: Dynamic milling process.

The feed direction of the tool is defined as the $x$ direction, and the coordinate direction is shown in Figure 4 . The $\phi_{j}$ shows the angular position of the tooth. The tangential and radial cutting forces of the tooth $j$ are expressed as $F_{t, j}$ and $F_{n, j}$; the angle between the radial cutting force and the vector cutting force is expressed as $\beta$. The transient cutting force of the $j$ th tooth of the end mill can be expressed as follows [11]:

$$
F_{x, j}=-K_{t} b g\left(\phi_{j}\right)\left(\begin{array}{c}
\Delta x\left(\sin \left(\phi_{j}\right) \cos \left(\phi_{j}\right)+K_{n} \sin ^{2}\left(\phi_{j}\right)\right) \\
+\Delta y\left(K_{n} \sin \left(\phi_{j}\right) \cos \left(\phi_{j}\right)+\cos ^{2}\left(\phi_{j}\right)\right)
\end{array}\right) \text {, }
$$

$$
F_{x, j}=K_{t} b g\left(\phi_{j}\right)\left(\begin{array}{c}
\Delta x\left(-K_{n} \cos \left(\phi_{j}\right) \sin \left(\phi_{j}\right)+\sin ^{2}\left(\phi_{j}\right)\right) \\
+\Delta y\left(\sin \left(\phi_{j}\right) \cos \left(\phi_{j}\right)-K_{n} \cos ^{2}\left(\phi_{j}\right)\right)
\end{array}\right) .
$$

The cutting force vector $F_{A, x}$ and $F_{A, y}$ of each of the cutter end mills with $N_{t}$ teeth in the $x$ and $y$ directions is 


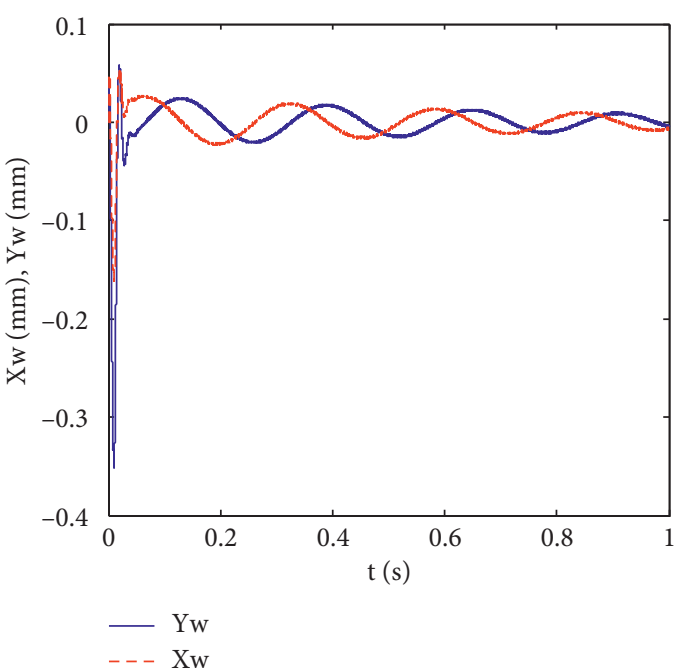

(a)

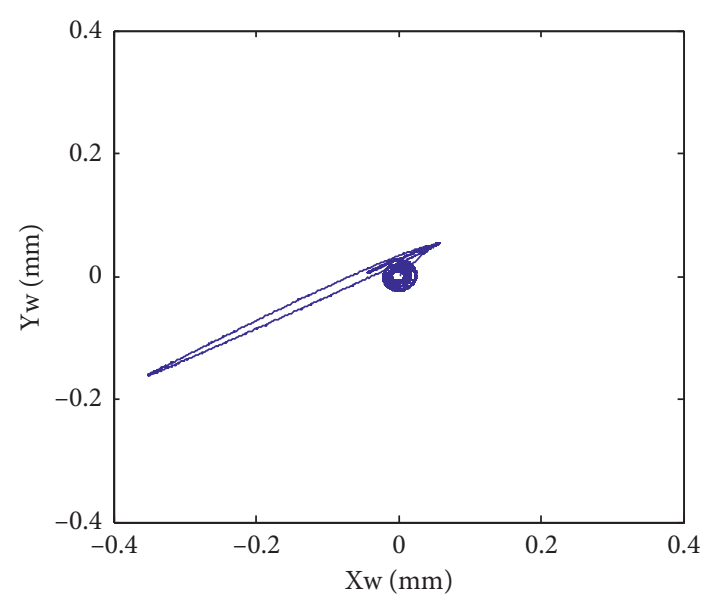

(b)

FIgURE 4: The displacement at the workpiece position based on the conventional PID: (a) displacement diagram in the $x$ and $y$ direction; (b) running track at the position of the workpiece.

$$
\begin{aligned}
& F_{A, x}=\sum_{j=1}^{N_{t}} F_{x, j}, \\
& F_{A, y}=\sum_{j=1}^{N_{t}} F_{y, j} .
\end{aligned}
$$

By putting equations (26) and (27) into equation (28), the cutting force expression shown in the following equation can be obtained:

$$
\begin{aligned}
& F_{A, x}=\frac{1}{2} b K_{t}\left(c_{x x} \Delta x_{i}+c_{x y} \Delta y_{i}\right), \\
& F_{A, x}=\frac{1}{2} b K_{t}\left(c_{y x} \Delta x_{i}+c_{y y} \Delta y_{i}\right),
\end{aligned}
$$

where

$$
\begin{aligned}
& c_{x x}=\left.\frac{1}{2}\left[\cos (2 \varphi)-2 K_{n} \varphi+K_{n} S\right]\right|_{\varphi_{s}} ^{\varphi_{e}}, \\
& c_{x y}=-\left.\frac{1}{2}\left[\sin (2 \varphi)+2 \varphi-K_{n} \cos (2 \varphi)\right]\right|_{\varphi_{s}} ^{\varphi_{e}}, \\
& c_{y x}=-\left.\frac{1}{2}\left[\sin (2 \varphi)-2 \varphi-K_{n} \cos (2 \varphi)\right]\right|_{\varphi_{s}} ^{\varphi_{e}}, \\
& c_{y y}=\left.\frac{1}{2}\left[\cos (2 \varphi)+2 K_{n} \varphi+K_{n} \sin (2 \varphi)\right]\right|_{\varphi_{s}} ^{\varphi_{e}},
\end{aligned}
$$

where $K_{t}$ is the tangential milling stiffness, $K_{n}$ is the radial milling stiffness, $b$ is the milling width, and $\Delta x_{i}, \Delta y_{i}$ is the dynamic depth of cut in the $x$ and $y$ directions, and the start and end angles of the tooth action are expressed as $\phi_{s}$ and $\phi_{e}$, respectively.

4.2. Stability Analysis. When the stability of the active magnetic bearing cutting system is analyzed, it is mainly about the dynamic displacement change of the workpiece end; however, there is the gap between the workpiece mounting position and the position of the electromagnetic bearing $B$, and the control force of the electromagnetic bearing is acted on the position of the electromagnetic bearing. In order to reduce stability analysis error, the displacement at the workpiece is corrected by the correction factor $k_{w}$, that is, $x_{c w}=k_{w} x_{m B}$. Therefore, the first step is to use the displacement of the electromagnetic bearing to indicate the following:

$$
q=\left[\begin{array}{c}
\theta_{y} \\
x \\
\theta_{x} \\
y
\end{array}\right]=\left[\begin{array}{cccc}
\frac{1}{L} & -\frac{1}{L} & 0 & 0 \\
\frac{l_{b}}{L} & \frac{l_{a}}{L} & 0 & 0 \\
0 & 0 & -\frac{1}{L} & \frac{1}{L} \\
0 & 0 & \frac{l_{b}}{L} & \frac{l_{a}}{L}
\end{array}\right] \cdot\left[\begin{array}{c}
x_{b A} \\
x_{b B} \\
y_{b A} \\
y_{b B}
\end{array}\right]=T_{s} q_{s} .
$$

Putting equation (31) into equation (23), the motion equation of the magnetic suspension rigid electric spindle rotor system can be obtained in magnetic bearing $B$ coordinates as

$$
M T_{s} \ddot{q}_{s}+(G+D) T_{s} \dot{q}_{s}+T_{s} P q_{s}=0 .
$$

If $\Delta e=\left[k_{w} \Delta x_{m B}, \Delta y_{m B}\right]^{T}$ is the dynamic cutting depth of milling, equation (35) can be obtained by putting equation (29) into equation (32):

$$
M T_{s}\{\Delta \ddot{e}\}+(G+D) T_{s}\{\Delta \dot{e}\}+T_{s} P\{\Delta e\}=\frac{1}{2} b K_{t} \frac{N_{t}}{2 \pi} \mathrm{H} \Delta e .
$$


TABLE 1: Electric spindle parameters.

\begin{tabular}{lcc}
\hline$m$ & Rotor mass & $25.8 \mathrm{~kg}$ \\
$l_{a}$ & Radial electromagnet center distance & $150 \mathrm{~mm}^{2}$ \\
$J_{x}, J_{y}$ & Moment of inertia of the rotor around the $x$-axis & $0.2251 \mathrm{kgm}^{2}$ \\
$J_{z}$ & Moment of inertia of the rotor around the $z$-axis & $0.3388 \mathrm{kgm}^{2}$ \\
$\delta$ & Radial electromagnetic bearing ideal air gap & $0.4 \times 10^{-3} \mathrm{~m}$ \\
$R_{s}$ & Spindle radius & $50 \mathrm{~mm}$ \\
$k_{i}$ & Current stiffness coefficient & $37.7 \mathrm{~N} / \mathrm{A}$ \\
$k_{s}$ & Displacement stiffness coefficient & $15.08 \mathrm{e} 4 \mathrm{~N} / \mathrm{m}$ \\
\hline
\end{tabular}

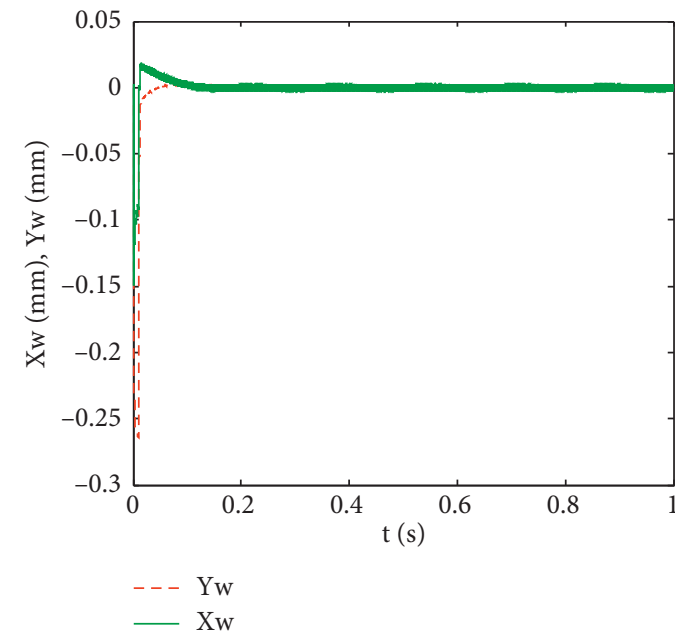

(a)

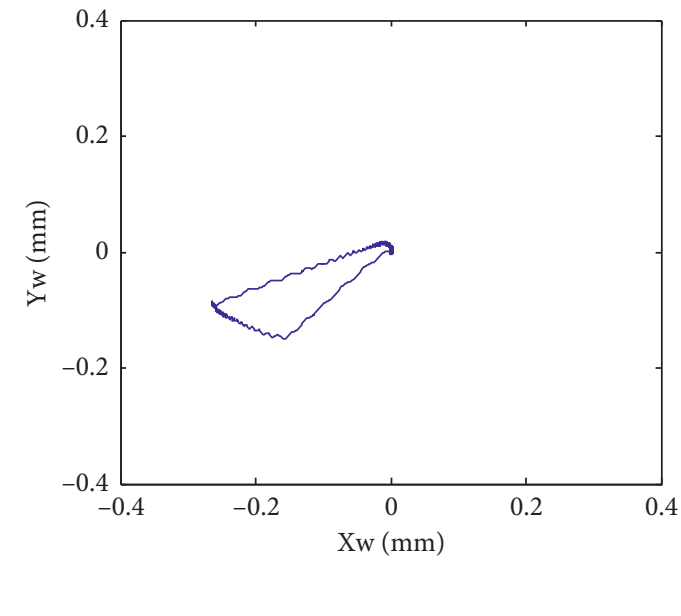

(b)

FIGURE 5: The displacement at the workpiece position based on modal decoupling: stability area of milling (a) without control and (b) with modal decoupling control.

The dimension of the matrix $H$ is $2 \times 2$, and the proportional coefficient is $c_{x x}, c_{x y}, c_{y x}, c_{y y}$.

In order to analyze the effect of the gyro effect of the active magnetic bearing on the region of the milled stability, convert equation (33) as follows [14]:

$$
\begin{aligned}
&\{-\left.M T_{s} \omega_{c}^{2}+(G+D) T_{s} \omega_{c}+T_{s} P\right\}\left\{\Delta e\left(i \omega_{c}\right)\right\}=\frac{N_{t}}{4 \pi} b K_{t} H \\
& \cdot\left(1-e^{-i \omega_{c} T}\right)\left\{\Delta e\left(i \omega_{c}\right)\right\} .
\end{aligned}
$$

If $\Gamma\left(i \omega_{c}\right)=1 /\left\{-M T_{s} \omega_{c}^{2}+(G+D) T_{s} \omega_{c}+T_{s} P\right\}$, then equation (34) is arranged out to obtain the closed-loop dynamic milling characteristic equation:

$$
\left\{I-\frac{N_{t}}{4 \pi} b K_{t}\left(1-e^{-i \omega_{c} T}\right)[B] \Gamma\left(i \omega_{c}\right)\right\}\left\{\Delta e\left(i \omega_{c}\right)\right\}=0 .
$$

If $\xi=-\left(N_{t} / 4 \pi\right) b K_{t}\left(1-e^{-i \omega_{c} T}\right)$, the eigenvalues of equation (35) are

$$
\xi=\xi_{\mathrm{Re}}+i \xi_{\mathrm{Im}} .
$$

When the real part of all the eigenvalues of equation (35) is less than zero, the milling process is stable. Otherwise, the milling process is unstable.
Equation (36) and $e^{-i \omega_{c} T}=\cos \left(\omega_{c} T\right)-i \sin \left(\omega_{c} T\right)$ are put into $\xi=-\left(N_{t} / 4 \pi\right) b K_{t}\left(1-e^{-i \omega_{c} T}\right)$, and the critical milling width $b_{\lim }$ is obtained:

$$
b_{\lim }=-\frac{4 \pi}{N_{t} K_{t}}\left\{\left\{\xi_{\mathrm{Re}}+i \xi_{\mathrm{Im}}\right\}+\frac{\xi_{\mathrm{Im}} \sin \left(\omega_{c} T\right)\left\{-i \xi_{\mathrm{Re}}+\xi_{\mathrm{Im}}\right\}}{1-\cos \left(\omega_{c} T\right)}\right\} \text {. }
$$

Make the imaginary part zero:

$$
\zeta=\frac{\xi_{\mathrm{Im}}}{\xi_{\mathrm{Re}}}=\frac{\sin \left(\omega_{c} T\right)}{1-\cos \left(\omega_{c} T\right)} .
$$

Equations (38) and (37) are arranged:

$$
b_{\lim }=-\frac{2 \pi}{N_{t} K_{t}} \xi_{\mathrm{Re}}\left(1+\xi^{2}\right),
$$

where $T=\left(\pi-2 \tan ^{-1} \xi+2 N \pi\right) / \omega_{c}$ is the number of ripples between the two teeth and $\omega_{c}$ chatter frequency.

\section{Milling System Stability Area Analyses}

The influence of the traditional PID controller and modal decoupling control on the displacement of the workpiece at the milling position is analyzed by simulation. The influence of the gyro effect on the milling stability region is analyzed. The parameters of the electric spindle rotor system used in 


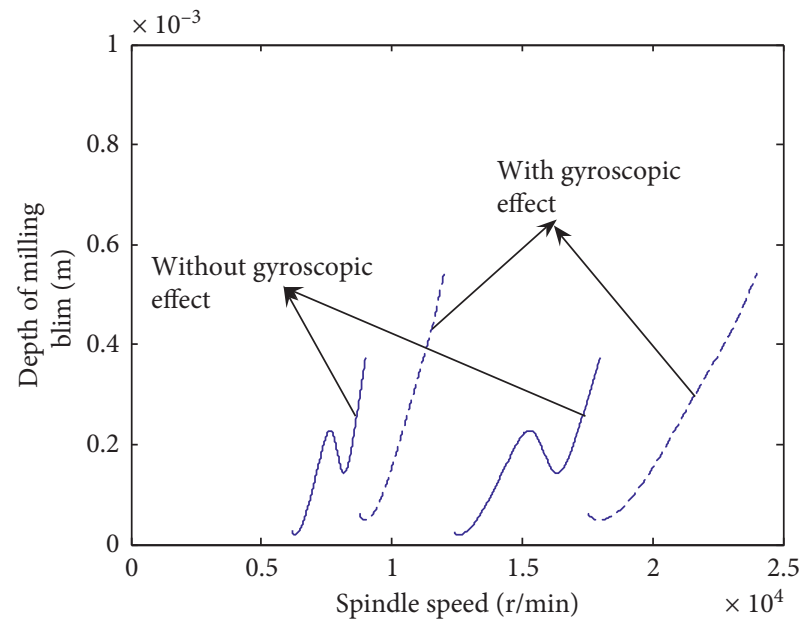

(a)

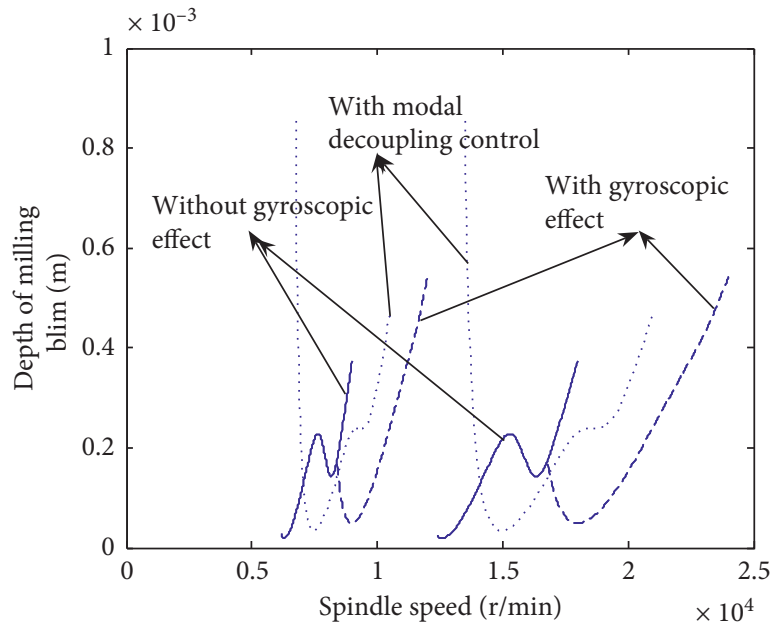

(b)

FIGURE 6: Effect of modal decoupling control on the stability of milling: stability area of milling (a) without control and (b) with modal decoupling control.

the simulation are shown in Table 1, and the milling parameters are $N_{4}=4$ and $K_{t}=2173 \mathrm{~N} / \mathrm{mm}^{2}$.

Figure 4 shows the displacement track at the workpiece position under the PID controller. It can be seen from Figure 4 that the rotor of the electric spindle cannot be stably suspended at the operation starts. The PID controller is turned on at $0.02 \mathrm{~s}$, and the electric spindle rotor gradually stabilizes under the action of the controller. The displacement in the $x$ and $y$ directions of the workpiece position gradually decreases, but there is fluctuation, which is due to the influence of the gyro cross-effect of the electric spindle rotor system. The modal of the electric spindle rotor is decoupled on the basis of the PID controller, and the rotational mode and the translational modal of the rotor system of the electric spindle are independently controlled. It can be seen from Figure 5 that the modal decoupling control can make the displacement of the workpiece position rapidly reduced and stable. Figure 5(a) shows when the modal decoupling control is also turned on at $0.02 \mathrm{~s}$, the displacement at the workpiece position quickly approaches zero (stable), and the fluctuations existing in the conventional PID control are overcome.

Figure 6 shows the effect of gyro effect on the stability of milling at different speeds: $n=6000 \mathrm{r} / \mathrm{min}$ and $n=15000 \mathrm{r} /$ min; the influence of gyro effect on the stability of milling is analyzed.

It can be seen from Figure 6(a) that the gyro effect will shift the milling stability to the right and move it slightly upward. The higher the speed, the more the right shifts, which indicate that the gyro effect makes the frequency of the critical stability zone of milling frequency value increase. And the higher the speed of the electric spindle rotor, the higher the frequency of the critical stability zone of the milling. This is because the gyro effect is related to the speed of the electric spindle, and as the rotor speed of the electric spindle increases, the gyro effect becomes more obvious. The modal decoupling control can greatly reduce the gyro effect on the critical stability zone of milling, which is shown in Figure 6(b).

\section{Conclusions}

Based on the mathematical model of electric spindle rotor system with the active magnetic bearing, the influence of the traditional PID controller and the modal decoupling control on the displacement of the milling workpiece position are analyzed. The displacement at the workpiece position quickly approaches zero (stable) with the modal decoupling controlling, and the fluctuations existing in the traditional PID control are overcome.

The effect of the gyro effect on the stability of milling at different rotor speeds of the electric spindle is analyzed. The simulation results show that the gyro effect can shift the stability area of the milling to the right. And this effect will be more obvious as the rotor speed of the electric spindle increases. The proposed modal decoupling control can reduce the influence of gyro effect on the stability of milling by separately adjusting the parameters of the translational mode and the rotational mode.

\section{Data Availability}

The data used to support the findings of this study are included within the article.

\section{Conflicts of Interest}

The authors declare that they have no conflicts of interest.

\section{Acknowledgments}

This research was supported by the National Natural Science Foundation of China (51505296) and Zhejiang Provincial Public Foundation of China (LGG19E050014). 


\section{References}

[1] C. Yue, H. Gao, X. Liu, S. Y. Liang, and L. Wang, "A review of chatter vibration research in milling," Chinese Journal of Aeronautics, vol. 32, no. 2, pp. 215-242.

[2] S. Sivrioglu and K. Nonami, "Sliding mode control with timevarying hyperplane for AMB systems," IEEE/ASME Transactions on Mechatronics, vol. 3, no. 1, pp. 51-59, 1998.

[3] A. Mystkowski, "Sensitivity and stability analysis of musynthesis AMB flexible rotor," Solid State Phenomena, vol. 164, no. 133, pp. 313-318, 2010.

[4] S. Sivrioglu and K. Nonami, "An experimental evaluation of robust gain-scheduled controllers for AMB system with gyroscopic rotor," in Proceedings of the 6th International Symposium on Magnetic Bearings, pp. 352-361, Cambridge, MA, USA, August 1998.

[5] Y. C. Zhang, G. J. Sun, and Y. J. Zhang, "Experimental verification for zero power control of $0.5 \mathrm{kwh}$ class flywheel system using magnetic bearing with gyroscopic effect," in Proceedings of the 1st International Conference on Machine Learning and Cybernetics, pp. 2059-2062, Beijing, China, November 2002.

[6] P. Efrain, B. Douglas, and K. Edson, "Vibration control using active magnetic actuators with the LQR control technique," in Proceedings of the 7th Brazilian Conference on Dynamics, Control and Applications, pp. 1-6, Presidente Prudente, Brazil, May 2008.

[7] K. Zhang and X. Dai, "Dynamic analysis and control of an energy storage flywheel rotor with active magnetic bearings," in Proceedings of the 2010 International Conference on Digital Manufacturing \& Automation, pp. 573-576, Changsha, China, 2010.

[8] B. Wang and Z. Deng, "Analysis of cross feedback control for the magnetically suspended flywheel rotor," in Proceedings of the 12th International Symposium on Magnetic Bearings, pp. 567-572, Nanjing, China, 2010.

[9] K. Zheng, L. Zhao, and H. Zhao, "Application of magnetic force lead control on a flywheel suspended by AMS," Chinese Journal of Mechanical Engineering, vol. 40, no. 7, pp. 175-179, 2004.

[10] K. Jiang, "Active magnetic bearing-rotor vibration control technology," Ph.D. Zhejiang University, Zhejiang, China, 2011.

[11] X. Qiao and C. Zhu, "Active control of milling chatter based on the built-in force actuator," Journal of Mechanical Engineering, vol. 1, no. 48, pp. 187-192, 2012.

[12] R. Siegwart, R. Larsouneur, and A. Traxler, "Design and performance of a high speed milling spindle in digital controlled active magnetic bearings," in Proceedings of the 2nd International Symposium on Magnetic Bearings, University of Tokyo, Tokyo, Japan, pp. 197-204, 1990.

[13] Q. Zhang, "Research on the suppression of gyroscopic effect of active magnetic bearing-flywheel energy storage system," 2012.

[14] Z. Li and Q. Liu, "Modeling and simulation of chatter stability for circular milling," Journal of Mechanical Engineering, vol. 46, no. 7, pp. 181-186, 2010. 